\title{
tDNA locus polymorphism and ecto-chromosomal DNA insertion hot-spots are related to the phylogenetic group of Escherichia coli strains
}

\author{
Correspondence \\ Pierre Germon \\ Pierre.Germon@tours.inra.fr
}

Received 29 September 2006

Revised 9 November 2006

Accepted 20 November 2006

\author{
Pierre Germon, ${ }^{1}$ David Roche, ${ }^{1}$ Sandrine Melo, ${ }^{1}$ \\ Sandrine Mignon-Grasteau, ${ }^{2}$ Ulrich Dobrindt, ${ }^{3}$ Jörg Hacker, ${ }^{3}$ \\ Catherine Schouler ${ }^{1}$ and Maryvonne Moulin-Schouleur ${ }^{1}$ \\ ${ }^{1}$ INRA - Centre de Tours, UR 1282 IASP, Pathogénie Bactérienne, 37380 Nouzilly, France \\ ${ }^{2}$ INRA - Centre de Tours, Station de Recherche Avicole, 37380 Nouzilly, France \\ ${ }^{3}$ Institut für Molekulare Infektionsbiologie, Universität Würzburg, Röntgenring 11, 97070 \\ Würzburg, Germany
}

\section{INTRODUCTION}

Escherichia coli is a very diverse species including both commensal and pathogenic strains. Pathogenic E. colistrains have been classified into intestinal or extra-intestinal pathogens according to their phenotypes and origin (Kaper et al., 2004). Among these extraintestinal pathogens are strains responsible for diseases in humans, such as uropathogenic E. coli (UPEC) or newborn meningitis E. coli (NMEC) strains, but also in avian species, such as avian pathogenic E. coli (APEC) strains (Ron, 2006).

The diversity of the E. coli species has been studied in great detail and six major phylogenetic groups (called ECOR groups A, B1, B2, C, D and E) have been identified (EscobarParamo et al., 2004; Lecointre et al., 1998; Ochman \&

Abbreviations: APEC, avian pathogenic E. coli; EAEC, enteroaggregative E. coli; ECDNA, ecto-chromosomal DNA; EHEC, enterohaemorrhagic E. coli; EIEC, enteroinvasive E. coli; ETEC, enterotoxigenic E. coli; EPEC, enteropathogenic E. coli; ExPEC, extra-intestinal pathogenic $E$. coli of human origin; IPEC, intestinal pathogenic $E$. coli; MFCA, multiple factorial analysis of correspondences; NMEC, newborn meningitis E. coli; NP, non-pathogenic E. coli; UPEC, uropathogenic E. coli.

Three supplementary tables and two supplementary figures are available with the online version of this paper.
Selander, 1984; Selander et al., 1987). This diversity of E. coli is closely related to the plasticity of the E. coli genome, whereby a strain can acquire or lose a specific function through horizontal gene transfers or mutational events (Hacker et al., 2003). From genome sequence data one can define a 'core' gene pool containing the genetic information required for essential cellular functions and a 'flexible' gene pool made of ecto-chromosomal DNA (ECDNA) and conferring upon the bacteria more specific traits necessary to adapt to certain niches (Dobrindt et al., 2004; Hayashi et al., 2001; Welch et al., 2002). ECDNA regions, such as genomic islands, may confer various new properties including pathogenicity, antibiotic resistance and fitness traits (Dobrindt et al., 2004; Dozois \& Curtiss, 1999) and are frequently found downstream of tDNA loci (DNA encoding tRNA - for a list of tDNA-associated genomic islands in $E$. coli strains see Supplementary Table S1, available with the online version of this paper).

Among the numerous tDNAs present in the genome of $E$. coli only a few are currently known to be targeted by ECDNA insertions (see Table S1) (Blattner et al., 1997; Hayashi et al., 2001). One can therefore ask whether all tDNA loci are potential targets for ECDNA insertions. This study reports a PCR-based screening of the integrity of a subset of tDNA loci in the genome of a collection of extraintestinal 
pathogenic E. coli strains of avian (APEC) or human (ExPEC) origin as well as on a subset of intestinal pathogenic E. coli (IPEC) or faecal isolates.

\section{METHODS}

Strains and growth conditions. Strains used in this study are described in Supplementary Table S2. To get information on potential ECDNA regions present in specific isolates an emphasis was placed on APEC and, to a lesser extent, on ExPEC strains. IPEC and faecal isolates were also included in order to unravel any very significant difference from other isolates. A significant number of strains from each of the four major phylogenetic groups are present in our subset. APEC strains were collected in France, Spain and Belgium between 1991 and 1999 (EU contract FAIR6 98-4093) (Stordeur et al., 2002). They were isolated from birds harbouring colibacillosis lesions and were confirmed to be pathogenic using the day-old chick model (Dho \& Lafont, 1984). All 22 APEC strains were epidemiologically unrelated (Stordeur et al., 2002). The ExPEC and IPEC isolates analysed in this study as well as faecal isolates and strains from the ECOR collection (E. coli reference collection) were from the strain collection of the Institut für Molekulare Infektionsbiologie, Universität Würzburg, and have been described before (Boyd \& Hartl, 1998; Dobrindt et al., 2001; Ochman \& Selander, 1984). IPEC isolates included in this study belonged to different pathotypes, with one enteropathogenic (EPEC), seven shiga-toxin producing E. coli including the EHEC strain EDL933, one enterotoxigenic (ETEC), one enteroaggregative (EAEC) and one enteroinvasive (EIEC) strain. Bacteria were grown in Luria-Bertani (LB) broth at $37^{\circ} \mathrm{C}$ with agitation unless otherwise stated.

Sequence analysis. Sequences of the entire genome of E. coli K-12 strain MG1655, the uropathogenic strain CFT073 and the enterohaemorrhagic strains Sakai and EDL933 (referred to in the text as MG1655, CFT073, Sakai and EDL933 respectively) were downloaded from the NCBI server (http://www.ncbi.nlm.nih.gov/) and analysed using the VectorNTI software (Invitrogen).

Chromosomal DNA extraction. Chromosomal DNA was purified from $1 \mathrm{ml}$ overnight culture using the QIAamp DNA mini kit (Qiagen). Chromosome quality was checked by $1 \%$ agarose gel electrophoresis.

Primers and PCR conditions used for tDNA screening. A subset of tDNA loci likely to be associated with ECDNA was selected out of the 85 tDNAs located in the E. coli core genome (except for ileY all the other 85 tDNA loci of strain MG1655 were also present in the genome of strains CFT073, Sakai and EDL933). Because of their stability and because their disruption might adversely affect the growth of strains or their adaptation to changing physiological conditions, tDNAs located inside rDNA (DNA corresponding to rRNA) operons were not studied further (Anton et al., 1998; Asai et al., 1999; Condon et al., 1995). Similarly, insertion inside polycistronic tDNA was considered unlikely since it might disturb the processing of the transcribed RNA into tRNA (King et al., 1986; Li \& Deutscher, 1996, 2002; Morl \& Marchfelder, 2001). For this reason, when dealing with polycistronic tDNAs, only the last tDNA was selected.

Primer pairs used in this study, and PCR conditions, are listed in Supplementary Table S3. Primers were designed so that their sequences were present in the genome of all four strains (MG1655, CFT073, EDL933 and Sakai). In all cases, one of the primers was located just $5^{\prime}$ of the tDNA analysed. The second primer was located downstream of the tDNA analysed in a region common to all four strains. pheV was the only exception since the design of the second primer would then have required amplification of DNA fragments larger than
$30 \mathrm{~kb}$ in the four sequenced strains and presumably in a significant number of the other tested strains. This situation has also been described by $\mathrm{Ou}$ et al. (2006). Primer pheV2 was therefore designed so that its sequence is present in strains MG1655 and CFT073 but not in strains EDL933 and Sakai. Because the order of the asnUVW tDNAs is not identical in the four strains (asnWUV in strains MG1655, EDL933 and Sakai, asnVUW in strain CFT073) one set of primers was designed to encompass the entire asn $U V W$ region and it was considered as a single locus in the rest of the study.

PCR reactions $(50 \mu \mathrm{l})$ were performed using an Applied Biosystems 9700 PCR apparatus and were set up in $1 \times$ buffer (ThermoPol buffer; New England Biolabs) using 1 U Taq DNA polymerase (New England Biolabs), $200 \mu \mathrm{M}$ of each dNTP, $0.8 \mu \mathrm{M}$ of each primer and $100 \mathrm{ng}$ of chromosomal DNA. Cycling conditions were as follows: 1 cycle of $5 \mathrm{~min}$ at $95^{\circ} \mathrm{C}, 30$ cycles of $30 \mathrm{~s}$ at $95^{\circ} \mathrm{C}, 30 \mathrm{~s}$ at $52^{\circ} \mathrm{C}$ and 1 min per kb at $72^{\circ} \mathrm{C}$, and a final extension of $5 \mathrm{~min}$ at $72^{\circ} \mathrm{C}$. PCR products were separated on $1 \%$ agarose gels for $1 \mathrm{~h}$ at $10 \mathrm{~V}$ per $\mathrm{cm}$ of gel.

For amplification of long PCR fragments, the LA PCR kit Ver2.1 (Takara) was used and cycling conditions were modified according to the manufacturer's instructions (see Table S3 for details).

Scoring scheme. An integrity score was calculated for each locus. A value of 0 was given for a negative PCR result and 1 for the presence of a PCR product, whatever its size. The integrity score of a locus was then calculated by summing the values obtained for this locus in all 54 strains.

ECOR grouping. Classification into phylogenetic groups A, B1, B2 and $\mathrm{D}$ was based on the amplification of genes $c h u A$ and $y j a A$, and of fragment TspE4C2.1, with three primer pairs: chuA1/chuA2, yjaA1/yjaA2 and TspE4C2.1/TspE4C2.2, as described by Clermont et al. (2000).

Statistical analysis. The probability of a tDNA locus being targeted by an ECDNA insertion was equal to $1 /$ (score of locus). When fragments of different size were observed at one locus, the distribution of these sizes was compared to the ECOR group of strains using a $\chi^{2}$ test and exact $P$-values (referred to as 'Size/ECOR group $P$-value') were calculated with the StatXact software (version 5.0, Cytel Software).

tDNA usage. Data concerning the codons recognized by one tRNA were collected from the E. coli MG1655 annotated genome (GenBank accession no. U00096). The frequency of each codon per thousand codons was calculated based on the frequency of codons in a set of 27 very highly expressed genes described by Sharp \& Li (1986). tDNA usage was then calculated by summing the frequency of the codons recognized by the corresponding tRNA.

Multiple factorial analysis of correspondences (MFCA) and hierarchical clustering. A factorial analysis of correspondences of the PCR results data was undertaken using the SPAD 6.0 software (Decisia, France). For each locus, the value corresponding to the approximate size of the PCR product was used; an absence of PCR product was given the value 0 . A modality was defined as a size in bp that a PCR product can take at one locus (e.g. asnUVW $=5300$ ). A strain and a modality were defined by their coordinates, the coordinates of a strain being its 34 modalities (i.e. the size of the PCR product obtained at each locus) and the coordinates of a modality being whether or not it is obtained for each of the 54 strains. The coordinates obtained for each strain and each modality were then transformed using matrices into new coordinates onto several factorial axes, each strain (or modality) having one coordinate on each factorial axis. For each factorial axis, using the coordinate of each strain (or modality) on this axis, a variance of the distribution of the strains (or modalities) was then calculated. Factorial axes were 
then ordered by decreasing level of variance, axis F1 being the axis for which the variance in the distribution of strains was the highest, F2 being the axis with the second highest variance, and so on.

Results were then represented graphically onto planes described by the F1/F2 axis and F1/F3 axis using the factorial coordinates of each modality. Data concerning the ECOR group and the pathotype were considered as supplementary observations and projected on the F1/F2 plane. From the factorial coordinates on the first five factorial axes obtained by the MFCA analysis, distances between strains were calculated and an ascending hierarchical clustering was performed using Ward's criteria (SPAD 6.0).

\section{RESULTS}

\section{Identification of tDNA targeted by ECDNA insertions}

Our analysis was restricted to a subset of 36 tDNAs belonging to the core genome, not located inside rDNA operons, and either monocistronic or the last tDNA of a polysictronic operon.

Complete results of the tDNA screening are summarized in Tables 1 and 2. Profiles obtained with strains MG1655 (line 11), CFT073 (line 33) and EDL933 (line 50) were consistent with the published sequences (Hayashi et al., 2001; Perna et al., 2001; Welch et al., 2002) (see Tables 1, 2 and S3).

Except for pheV, a positive PCR result was considered as reflecting the absence of insertion of ECDNA region. Conversely, we assumed that a negative PCR most likely indicated that primers were too far apart to allow any amplification in our conditions, thus suggesting the presence of an ECDNA region. The integrity score calculated for each locus was consequently an indication of how frequently this locus was a site for ECDNA insertions. Loci with a high score are presented in Table 1: actually, a majority of loci $(19 / 36)$ had high scores $(\geqslant 47)$. For these loci the probability that a negative PCR was observed was below $1 / 47$, i.e. $2.2 \%$. These loci are therefore unlikely to be targeted by ECDNA insertions.

Loci with a low score are presented in Table 2 and can be considered preferred sites for ECDNA insertions; the six loci with the lowest scores were asp $V$, pheV, leuX, thrW, metV and asnT. Our scoring scheme was validated by the fact that tDNA loci known as ECDNA insertion hot-spots such as leuX $($ score $=13), \operatorname{metV}($ score $=14), \operatorname{asn} T($ score $=17)$ and selC $($ score $=36)$ were given low scores (Redford \& Welch, 2002).

In most cases high scores were correlated with a low level of variability of the tDNA, with no more than two or three different sizes of PCR product. However, a high diversity in PCR product size was observed for loci with high scores such as $l y s Q$ (13 different sizes), $\operatorname{tyr} V$ (10 different sizes), $\operatorname{argQ}$ (9 different sizes) and leuV (5 different sizes) (see Supplementary Fig. S1 for examples). This suggests that these loci, while not being frequent sites of ECDNA insertions, are nevertheless highly variable regions. Such polymorphism is observed when comparing these same regions in strains MG1655, CFT073 and EDL933 (see Table S3).

\section{Polycistronic tDNAs have high integrity scores}

In order to explain why certain tDNAs were generally intact while others seemed to be frequently targeted by ECDNA insertions we investigated whether this could be related to codon usage, tDNA transcription rate or organization of the corresponding region. Indeed one hypothesis could be that tDNAs with the lowest scores encoded tRNAs recognizing rare codons or less transcribed tRNAs. For that purpose, a tDNA usage score was calculated based on data from Sharp \& Li (1986) (see Methods) and estimated transcription rate data from Ardell \& Kirsebom (2005) were used. According to our results highly transcribed tDNAs or tDNAs encoding tRNA recognizing frequently used codons (high tDNA usage) tend to have high integrity scores (Fig. 1a, b). However, we could not show a direct relationship between integrity score and either codon usage or tDNA transcription rate. More striking differences were seen when we compared the organization of the tDNAs (monocistronic vs polycistronic): we found that polycistronic tDNAs generally had high scores, indicating that they were unlikely targets for ECDNA insertion (Fig. 1b). Indeed, met $V$ was the only tDNA with a low score $($ score $=14)$ and a polycistronic organization (Fig. 1b, arrowhead). Interestingly, the highly variable high-integrity loci mentioned above (i.e. $\arg Q$, $l e u V$, lys $Q$ and $t y r V$ ) are all polycistronic loci.

\section{tDNA screening profiles reflect the diversity of E. coli}

We next wanted to know if the pattern of amplification at tDNA loci for a particular strain was related to either its pathotype or its ECOR group. A $\chi^{2}$ test was performed to compare the distribution of PCR product sizes between the four ECOR groups. For loci $l y s V, l y s Q, \operatorname{argQ}, t y r V, l e u V$, proL, leuZ, serW, selC, serX, asnUVW, glyU, asnT, serU, $\arg W, \arg U$ and $m e t V$ this distribution was very unlikely to be random $(P<0.001$ or $P<0.01)$. Therefore, strains from different ECOR groups are significantly different at several tDNA loci.

A multiple factorial analysis of correspondences (MFCA) was performed to unravel potential associations (Fig. 2). Figs 2(a) and 2(b) depict the distribution of the different PCR product sizes listed in Tables 1 and 2 in two factorial planes: plane F1/F2 defined by the two factorial axes F1 and F2, and plane F1/F3 defined by axes F1 and F3. Axes F1, F2 and F3 were the three most discriminating factorial axes, explaining respectively $12.14 \%, 7.96 \%$ and $6.22 \%$ of the distribution. In both Fig. 2(a) and Fig. 2(b), two modalities being close to each other indicates an association of these two modalities; the larger the symbols of these two modalitites, the stronger the association. For example, Fig. 2(a) indicates that a fragment of $2700 \mathrm{bp}$ at $g l y U$ is frequently 
Table 1. tDNA screening results (integrity score $\geqslant 47$ )

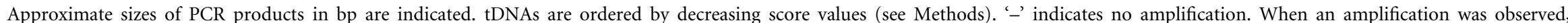

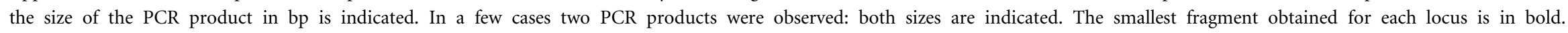



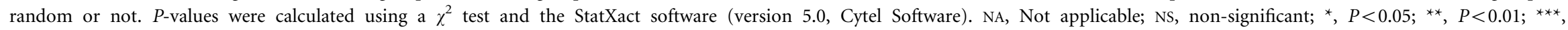
$P<0.0001$.

\begin{tabular}{|c|c|c|c|c|c|c|c|c|c|c|c|c|c|c|c|c|c|c|c|c|c|c|}
\hline & Strain & ECOR & Pathotype & valW & $t h r T$ & proM & proK & metY & lysV & lys $Q$ & $g l y Y$ & $\operatorname{asp} U$ & $\arg Q$ & alaX & $\operatorname{gln} X$ & leuU & ileX & $\operatorname{tyr} V$ & leuV & $\operatorname{ser} T$ & proL & leuZ \\
\hline 1 & BEN 0014 & A & APEC & 630 & 580 & 550 & 890 & 630 & 5000 & 1750 & 600 & 300 & 1450 & 630 & 600 & 690 & 1100 & 1000 & 1230 & 630 & 900 & 670 \\
\hline 2 & BEN 0278 & A & APEC & 630 & 580 & 550 & 890 & 630 & 5200 & 1700 & 600 & 300 & 1450 & 630 & 600 & 690 & 1100 & 1200 & 1230 & 630 & 900 & 670 \\
\hline 3 & BEN 1588 & A & APEC & 630 & 580 & 550 & 890 & 630 & 5200 & 1850 & 600 & 300 & 1450 & 630 & 600 & 690 & 1100 & 1200 & 1250 & 630 & 900 & - \\
\hline 4 & MT458 & A & APEC & 630 & 580 & 550 & 1050 & 630 & 5200 & 1750 & 600 & 300 & 1450 & 630 & 700 & 690 & - & 1000 & 1230 & 630 & 900 & 670 \\
\hline 5 & DPA065 & A & EAEC & 630 & 580 & 550 & 890 & 630 & 5000 & 1850 & 600 & 300 & 1450 & 630 & 600 & 690 & 1100 & 1200 & 1150 & 630 & - & 670 \\
\hline 6 & $5720 / 96$ & A & EHEC & 630 & 580 & 550 & 890 & 630 & 5000 & 1850 & 600 & 300 & 1450 & 630 & 600 & 690 & 1100 & 1200 & 1230 & 630 & 900 & 670 \\
\hline 7 & EDL1284 & A & EIEC & 630 & 580 & 550 & 890 & 630 & 5000 & 1330 & 600 & 300 & 1600 & 700 & 600 & 690 & 1100 & 2000 & - & 630 & 900 & 670 \\
\hline 8 & C9221a & A & ETEC & 630 & 580 & 550 & 890 & 630 & 5000 & 1330 & 600 & 300 & 1450 & 630 & 600 & 690 & 1100 & 1050 & 1250 & 630 & 900 & 670 \\
\hline 9 & ECOR1 & A & Faecal & 630 & 580 & 550 & 1050 & 630 & 5000 & 1850 & 600 & 300 & 1450 & 630 & 600 & 690 & 1100 & 1200 & 1250 & 630 & 900 & 670 \\
\hline 10 & ECOR25 & A & Faecal & 630 & 580 & 550 & 890 & 630 & 5000 & 1850 & 600 & 300 & 1450 & 630 & 600 & 690 & 1100 & 1200 & 1250 & 630 & 900 & 670 \\
\hline 11 & MG1655 & A & NP & 630 & 580 & 550 & 1050 & 630 & 5200 & 1850 & 600 & 300 & 1480 & 630 & 600 & 690 & 1100 & 1200 & 1230 & 630 & 900 & 670 \\
\hline 12 & $1639 / 77$ & B1 & EHEC & 630 & 580 & 550 & 890 & 630 & 5200 & 1750 & 600 & 300 & 1400 & 630 & 600 & 690 & 1100 & 870 & 1230 & - & - & 670 \\
\hline 13 & $4797 / 97$ & B1 & EHEC & 630 & 580 & 550 & 890 & 630 & 1000 & 1750 & 600 & 300 & 1450 & 630 & 600 & 690 & 1100 & 500 & 1230 & - & - & 670 \\
\hline 14 & ECOR28 & B1 & Faecal & 630 & 580 & 550 & 1050 & 630 & 1000 & 1750 & 600 & 300 & 1350 & 630 & 600 & 690 & 1100 & 870 & 1350 & 630 & 900 & 670 \\
\hline 15 & ECOR58 & B1 & Faecal & 630 & 580 & 550 & 1050 & 630 & 1000 & 1620 & 600 & 300 & 1450 & 630 & - & 690 & 1100 & 1000 & 1230 & 630 & 900 & 670 \\
\hline 16 & ECOR66 & B1 & Faecal & 630 & 580 & 550 & 890 & 630 & 5000 & 1330 & 600 & 300 & 1200 & 630 & 600 & 690 & 1100 & 1250 & 1150 & 630 & 250 & 670 \\
\hline 17 & BEN 0079 & B2 & APEC & 630 & 580 & 550 & 890 & 630 & 5000 & 1620 & 600 & 300 & 1200 & 630 & 600 & 690 & 1100 & 2000 & 1150 & 630 & 250 & 670 \\
\hline 18 & BEN 0098 & B2 & APEC & 630 & 580 & 550 & 890 & 630 & 5000 & 1150 & 600 & 300 & 1200 & 630 & 600 & 690 & 1100 & 2000 & 1230 & 630 & 250 & 670 \\
\hline 19 & BEN 0100 & B2 & APEC & 630 & 580 & 550 & 890 & 630 & 5000 & 1150 & 600 & 300 & 1200 & 630 & 600 & 690 & 1100 & 2000 & 1150 & 630 & 900 & 670 \\
\hline 20 & BEN 0134 & B2 & APEC & 630 & 580 & 550 & 890 & 630 & 5000 & 1330 & 1000 & 300 & 1200 & 500 & 600 & 690 & 1100 & 2000 & 1150 & 630 & 250 & 670 \\
\hline 21 & BEN 0277 & B2 & APEC & 630 & 580 & 550 & 890 & 630 & 5000 & 1330 & 600 & 300 & 1200 & 630 & 600 & 690 & 1100 & 2000 & - & 630 & 250 & 670 \\
\hline 22 & BEN 0288 & B2 & APEC & 630 & 580 & 550 & $880 / 1050$ & 630 & 5000 & 1330 & 600 & 300 & 1200 & 630 & 600 & 690 & 1100 & 2000 & - & 630 & 250 & 670 \\
\hline 23 & BEN 0338 & B2 & APEC & 630 & 580 & 550 & 1050 & 630 & 5000 & 1260 & 600 & 300 & 1050 & 630 & 600 & 690 & 1100 & 1850 & 1230 & 630 & 250 & 670 \\
\hline 24 & BEN 0371 & B2 & APEC & 630 & 580 & 550 & 890 & 630 & 5000 & 1330 & 600 & 300 & 1200 & 500 & 600 & 690 & 1100 & 2000 & 1150 & 630 & 250 & 670 \\
\hline 25 & BEN 0374 & B2 & APEC & 630 & 580 & 550 & 1050 & 630 & 5000 & 1330 & 600 & 300 & 1200 & 630 & 600 & 690 & 1100 & 2000 & 1150 & 630 & 250 & 670 \\
\hline 26 & BEN 0591 & B2 & APEC & 630 & 580 & 550 & 890 & 630 & 5200 & 1080 & 600 & 300 & 1200 & 630 & 600 & 690 & 1100 & 2000 & 1230 & - & 250 & 670 \\
\hline 27 & BEN 1567 & B2 & APEC & 630 & 580 & 550 & 890 & 630 & 5000 & 1330 & 600 & 300 & 1200 & 630 & 600 & 690 & - & 2000 & 1150 & 630 & 250 & 670 \\
\hline 28 & BEN 2908 & B2 & APEC & 630 & 580 & 550 & 890 & 630 & 5000 & 1470 & 600 & 300 & 1200 & 630 & 600 & 690 & 1100 & 1850 & 1150 & 630 & 250 & 670 \\
\hline 29 & MT 140 & B2 & APEC & 630 & 580 & 550 & 1050 & 630 & 5000 & 1470 & 600 & 300 & 1050 & 500 & 600 & 690 & 1100 & 1850 & 1150 & 630 & 250 & 670 \\
\hline 30 & E2348/69 & B2 & EPEC & 630 & 580 & 550 & 890 & 630 & 5000 & 1620 & 600 & 300 & 1100 & 630 & 600 & 690 & 1100 & - & 1250 & 630 & 250 & 670 \\
\hline 31 & 536 & B2 & ExPEC & 630 & 580 & 550 & 890 & 630 & 5000 & 1330 & 600 & 300 & 1600 & 700 & 600 & 690 & 1100 & 2000 & - & 630 & 250 & 670 \\
\hline 32 & ABU83972 & B2 & ExPEC & 630 & 580 & 550 & 890 & 630 & 5000 & 1300 & 600 & 300 & 1200 & 630 & 600 & 690 & 1100 & - & 1150 & 630 & - & 670 \\
\hline 33 & CFT073 & B2 & ExPEC & 630 & 580 & 550 & 890 & 630 & 5000 & 1310 & 600 & 300 & 1350 & 630 & 600 & 690 & 1100 & - & 1150 & 630 & 250 & 670 \\
\hline 34 & IHE3034 & B2 & ExPEC & 630 & 580 & 550 & 890 & 630 & 5000 & 1330 & 600 & 300 & 1200 & 630 & 600 & 690 & 1100 & 2000 & 1150 & 630 & 250 & 670 \\
\hline
\end{tabular}


Table 1. cont.

\begin{tabular}{|c|c|c|c|c|c|c|c|c|c|c|c|c|c|c|c|c|c|c|c|c|c|c|}
\hline & Strain & ECOR & Pathotype & valW & thrT & proM & proK & met $Y$ & lysV & lys $Q$ & $\operatorname{gly} Y$ & $\operatorname{asp} U$ & $\operatorname{argQ}$ & alaX & $g \ln X$ & leuU & ileX & tyr $V$ & $\operatorname{leu} V$ & ser $T$ & proL & leuZ \\
\hline 35 & J96 & B2 & ExPEC & 630 & 580 & 550 & 890 & 630 & 5000 & 1330 & 650 & 300 & 1600 & 700 & 600 & 690 & 1100 & 2000 & - & 630 & 250 & 670 \\
\hline 36 & P42 & B2 & ExPEC & 630 & 580 & 550 & 890 & 630 & 5000 & 1330 & 600 & 300 & 1450 & 630 & 600 & 690 & 1100 & - & 1150 & 630 & 250 & 670 \\
\hline 37 & RS218 & B2 & ExPEC & 630 & 580 & 550 & 890 & 630 & 5000 & 1330 & 600 & 300 & 1100 & 630 & 600 & 690 & 1100 & 2000 & 1150 & 630 & 250 & 670 \\
\hline 38 & W1825 & B2 & ExPEC & 630 & 580 & 550 & 890 & 630 & 5000 & 1330 & 600 & 300 & 1200 & 630 & 600 & 690 & 1100 & 2000 & 1150 & 630 & 250 & 670 \\
\hline 39 & 764 & B2 & Faecal & 630 & 580 & 550 & 890 & 630 & 5000 & 1330 & 600 & 300 & 1200 & 630 & 600 & 690 & 1100 & 2000 & 1150 & 630 & 250 & 670 \\
\hline 40 & ECOR51 & B2 & Faecal & 630 & 580 & 550 & 890 & 630 & 5000 & 1450 & 600 & 300 & 1200 & 630 & 600 & 690 & 1100 & - & 1150 & 630 & 250 & 670 \\
\hline 41 & F54 & B2 & Faecal & 630 & 580 & 550 & 890 & 630 & 5000 & 1330 & 600 & 300 & 1200 & 500 & 600 & 690 & 1100 & 1250 & 1150 & 630 & - & 670 \\
\hline 42 & BEN 0071 & $\mathrm{D}$ & APEC & 630 & 580 & 550 & 1050 & 630 & 5000 & 1470 & 600 & 300 & 1250 & 630 & 600 & 690 & 1100 & 1000 & 1150 & 630 & 900 & - \\
\hline 43 & BEN 0280 & $\mathrm{D}$ & APEC & 630 & 580 & 550 & 890 & 630 & 5000 & 1450 & 600 & 300 & 1200 & 630 & 600 & 690 & 1100 & 400 & 1250 & 630 & 900 & - \\
\hline 44 & BEN 0955 & $\mathrm{D}$ & APEC & 630 & 580 & 550 & 890 & 630 & 5200 & 1470 & 600 & 300 & 1450 & 630 & 600 & - & 1100 & 500 & 1150 & 630 & 250 & 670 \\
\hline 45 & BEN 0961 & $\mathrm{D}$ & APEC & 630 & 580 & 550 & 2300 & 630 & 5200 & 1470 & 600 & 300 & 1400 & 630 & 600 & - & 1100 & 500 & 1150 & 630 & 250 & 670 \\
\hline 46 & BEN 1189 & $\mathrm{D}$ & APEC & 630 & 580 & 550 & 2300 & 630 & 5200 & 1470 & 600 & 300 & 1100 & 630 & 600 & 690 & - & 500 & 1150 & 630 & 250 & 670 \\
\hline 47 & 493bi & $\mathrm{D}$ & EHEC & 630 & 580 & 550 & 890 & 630 & 4500 & 1260 & 600 & 300 & 1100 & 630 & 600 & 690 & 1100 & 500 & 900 & - & 900 & - \\
\hline 48 & $5714 / 96$ & $\mathrm{D}$ & EHEC & 630 & 580 & 550 & 890 & 630 & 5000 & 1620 & 600 & 300 & 1450 & 630 & 600 & 690 & 1100 & 870 & 1230 & - & 900 & - \\
\hline 49 & $86-24$ & $\mathrm{D}$ & EHEC & 630 & 580 & 550 & 890 & 630 & 1000 & 1310 & 600 & 300 & 1250 & 630 & 600 & 690 & 1100 & 1000 & 900 & - & 900 & 670 \\
\hline 50 & EDL933 & D & EHEC & 630 & 580 & 550 & 890 & 630 & 1000 & 1300 & 600 & 300 & 1250 & 500 & 600 & 690 & 1100 & 1000 & 900 & - & 900 & - \\
\hline 51 & ECOR40 & D & ExPEC & 630 & 580 & 550 & 890 & 630 & 5000 & 1310 & 600 & 300 & 1100 & 630 & 700 & 690 & 1100 & 400 & 1150 & 630 & - & 670 \\
\hline 52 & ECOR50 & $\mathrm{D}$ & ExPEC & 630 & 580 & 550 & 890 & 630 & 5000 & 1670 & 600 & 300 & 1250 & 630 & 600 & 690 & - & 2000 & - & 630 & 900 & - \\
\hline 53 & ECOR31 & $\mathrm{D}$ & Faecal & 630 & 580 & 550 & 890 & 630 & 1200 & 1470 & 600 & 300 & 1350 & 630 & 600 & 690 & 1100 & 830 & 1230 & 630 & 900 & 670 \\
\hline 54 & ECOR42 & $\mathrm{D}$ & Faecal & 630 & 580 & 550 & 890 & 630 & 1400 & 1620 & 600 & 300 & 1400 & 630 & 600 & 690 & 1100 & 500 & 1230 & 630 & - & 670 \\
\hline & Score & & & 54 & 54 & 54 & 54 & 54 & 54 & 54 & 54 & 54 & 54 & 54 & 53 & 52 & 50 & 49 & 48 & 47 & 47 & 47 \\
\hline & $\begin{array}{l}\text { Size/ECOR } \\
\text { group } P \text {-value }\end{array}$ & & & NA & NA & $\mathrm{NA}$ & NS & NA & $* * *$ & $* * *$ & NS & NA & $* * *$ & NS & * & NS & NS & $* * *$ & $* * *$ & * & $* * *$ & $\star * *$ \\
\hline
\end{tabular}




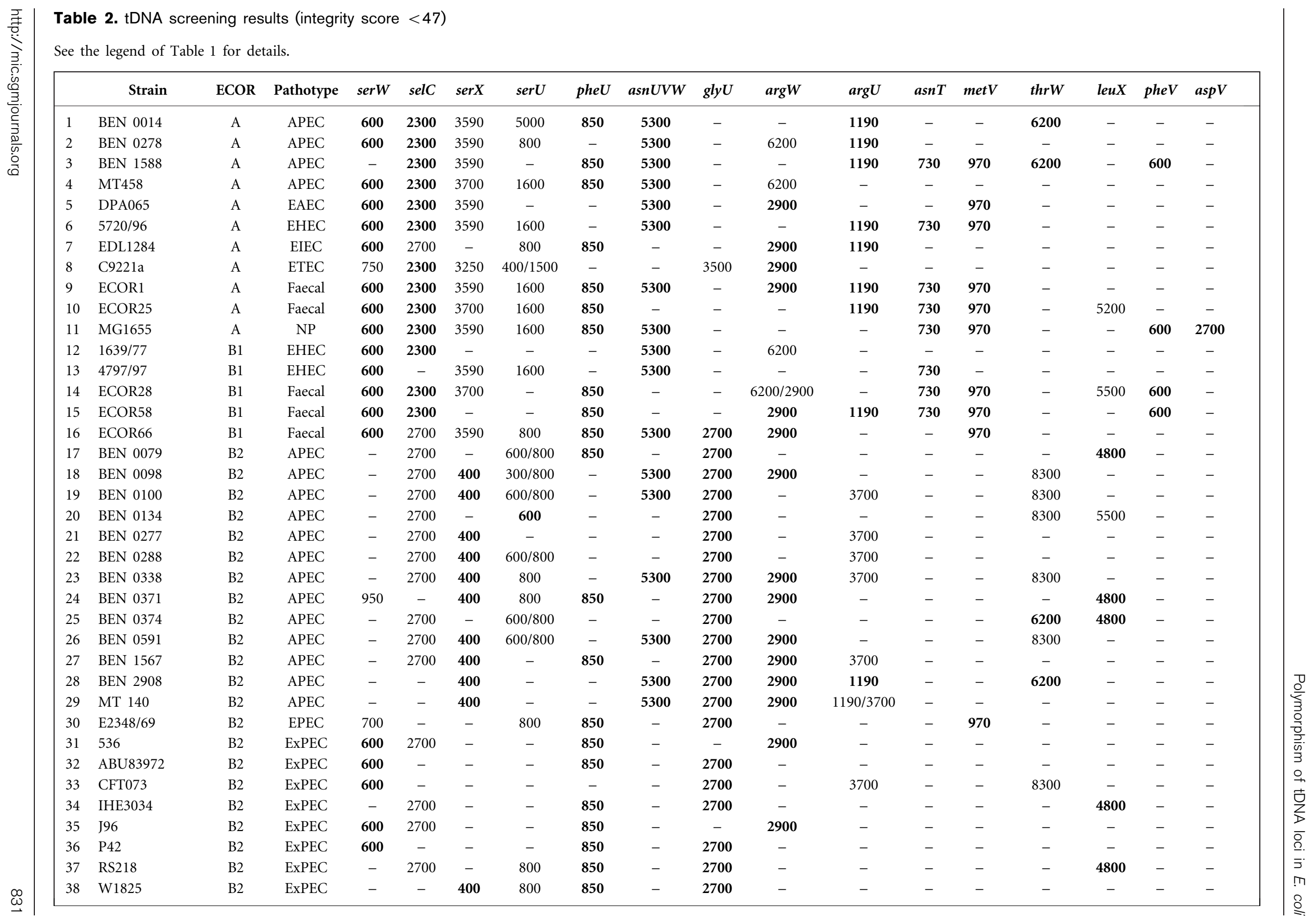




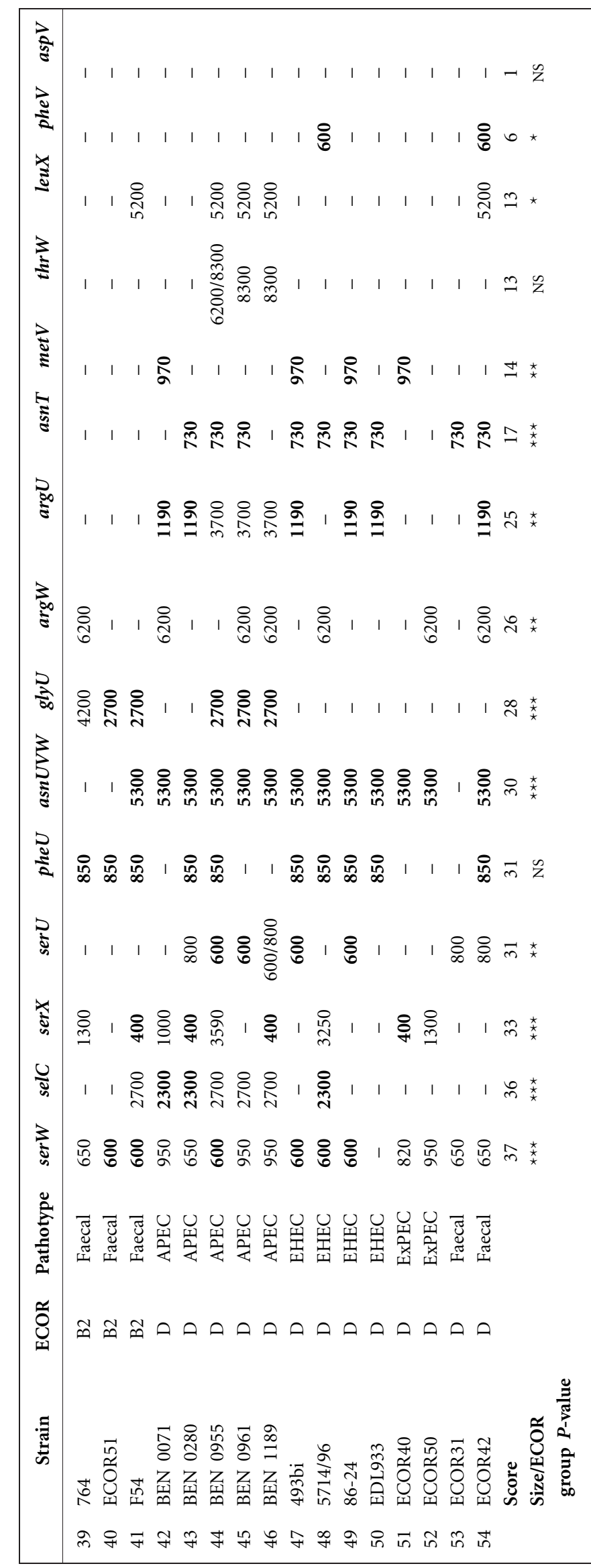

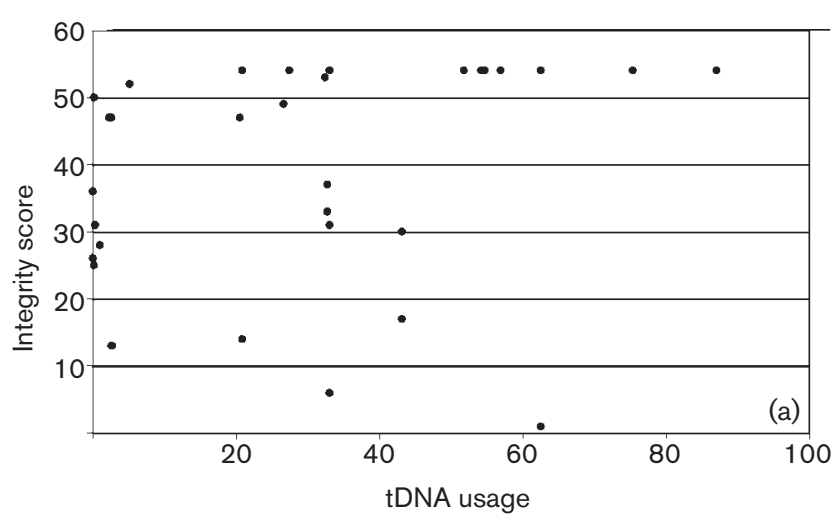

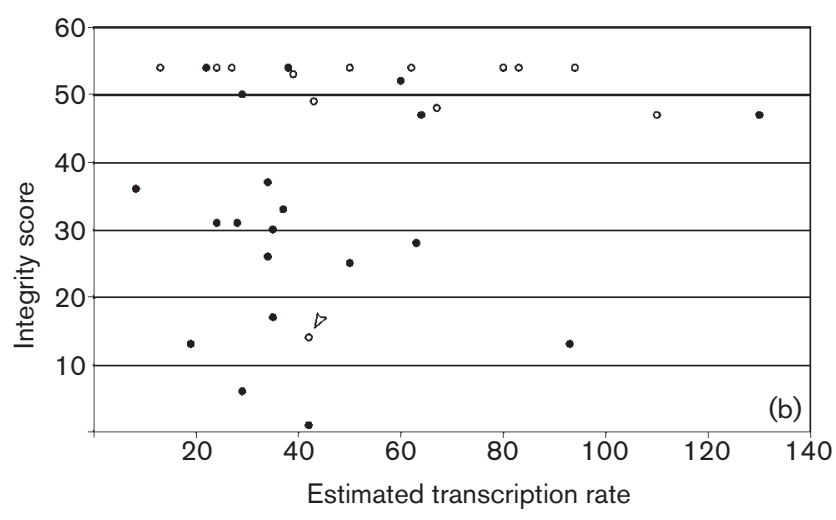

Fig. 1. Highly transcribed and/or polycistronic tDNAs frequently have high integrity scores. Correlations between integrity score and tDNA usage (a) or transcription rate (b) are shown. In (b), polycistronic tDNAs are shown as open symbols, monocistronic tDNAs as closed symbols. The only polycistronic tDNA locus with a low integrity score (metV) is indicated by an open arrowhead. tDNA usage was calculated as described in Methods using data from Sharp \& $\mathrm{Li}$ (1986). Transcription rate data were from Ardell \& Kirsebom (2005).

associated with a fragment of $2700 \mathrm{bp}$ at $s e l C$, of $400 \mathrm{bp}$ at ser $X$ and of $2000 \mathrm{bp}$ at $\operatorname{tyrV}$ (Fig. 2a, circled area). From this analysis, it is can be concluded that certain associations, such as that mentioned above, are favoured.

The ECOR group of strains was then superimposed upon the distribution of modalities. This indicated that strains from different ECOR groups were associated with different combinations of PCR product sizes: the four PCR products described above were associated with strains of the B2 group. Similarly, fragments of $500 \mathrm{bp}$ at $t y r V, 5200 \mathrm{bp}$ at $l y s V, 6200 \mathrm{bp}$ at $\arg W$ and $5300 \mathrm{bp}$ at $a s n U V W$ were more frequently associated with strains of ECOR group D. Strains of ECOR groups A and B1 were not clearly separated in the F1/F2 plane. However, projection on the F1/F3 plane unravelled additional associations that allowed the distinction of strains from ECOR groups A and B1. Indeed, in contrast to strains of the B1 group, group A strains were 

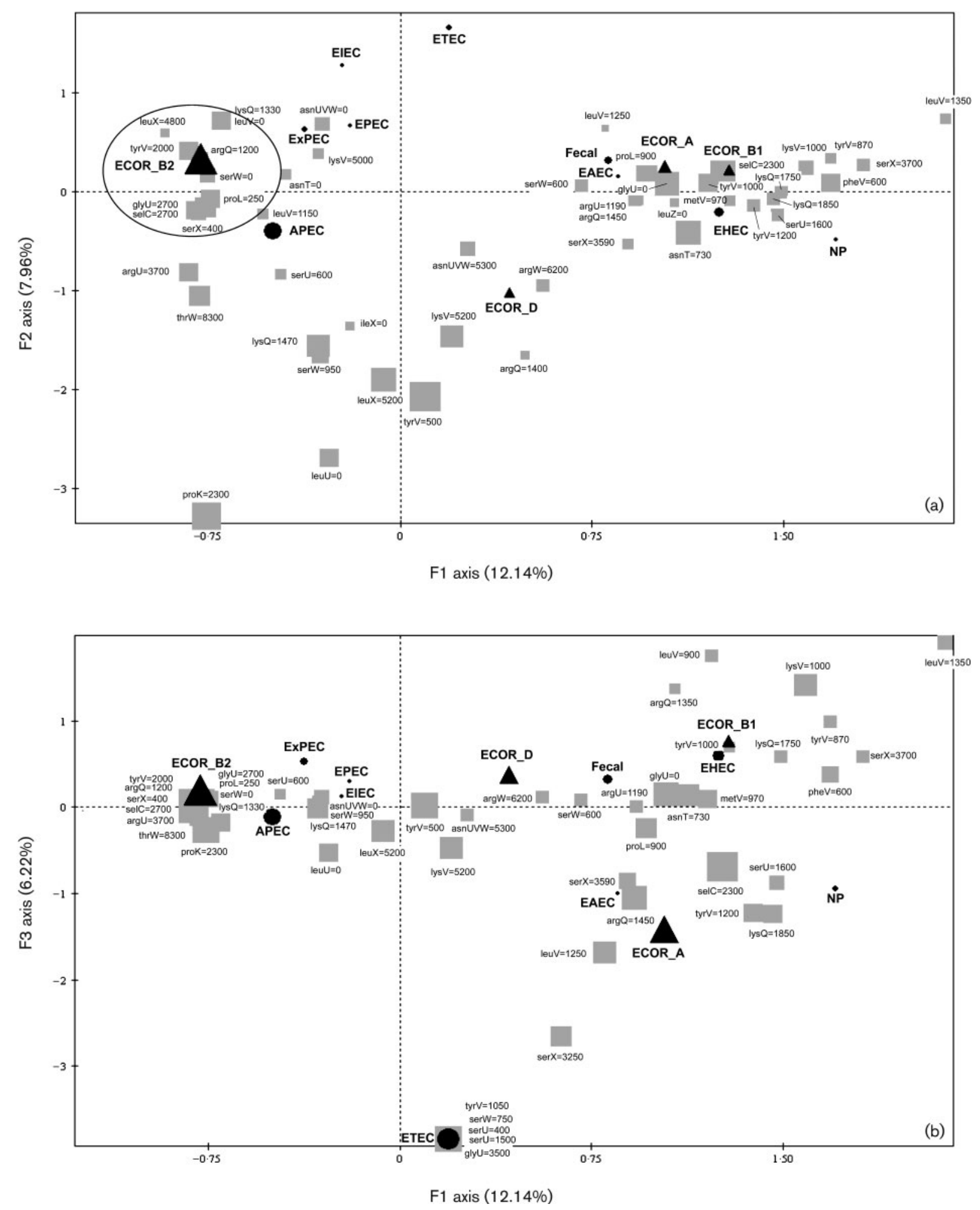

Fig. 2. Graphical representation of the results of the MFCA analysis. MFCA analysis was performed using the SPAD software. A modality was defined as a size in bp that a PCR product can take at one locus (e.g. asnUVW=5300). See Methods for details. The distribution of modalities was represented graphically onto planes described by the F1/F2 axis (a) and F1/F3 axis (b). The size of the squares is proportional to the contribution of the modality to the variance of the axis: the bigger the square, the more this modality contributes to the variance of the axis. Data concerning the ECOR group (black circles) and the pathotype (black triangles) were considered supplementary observations and projected on the F1/F2 and F1/F3 planes. The size of the circles and triangles is proportional to how well the distribution of these ECOR groups or pathotypes is explained by the projection on the F1/F2 or F1/F3 planes. 




associated with fragments of $1450 \mathrm{bp}$ at $\operatorname{argQ,} 1250 \mathrm{bp}$ at leuV, 1850 bp at lysQ, 1200 bp at tyrV and 2300 bp at selC.

We also investigated whether the distribution of tDNA PCR product sizes was related to the pathotype of strains.
Fig. 3. Dendrogram obtained after ascending hierarchical classification of strains. Factorial coordinates on the five main axes resulting from the MFCA analysis were used to calculate distances between strains and cluster strains using Ward's criteria. The distance scale is shown at the bottom of the figure. The four classes that could be distinguished at a distance of 0.109 are represented in the first column at the right of the dendrogram. The phylogenetic (ECOR) group and the pathotype of strains are given in the second and third columns, respectively, at the right of the dendrogram.

Interesting differences were seen between APEC and human ExPEC strains: most B2 strains showing no amplification at ser $W$ were APEC strains. Similarly, most strains yielding a 2700 bp product at selC and a $400 \mathrm{bp}$ fragment at ser $X$ were APEC strains. However, the small number of strains in the other pathotypes limited the conclusions that could be made concerning these pathotypes.

From the MFCA analysis, an ascending hierarchical clustering was then performed (Fig. 3). Interestingly, the clustering we obtained overlapped quite well with the ECOR clusters. Strains were separated into four major clusters: cluster 1 contained mostly group B2 strains and cluster 4 group A strains, cluster 2 contained only APEC strains belonging to both the $\mathrm{D}$ and $\mathrm{B} 2$ groups, while cluster 3 was the most heterogeneous, containing A, B1 and D strains. Interestingly, cluster 2 was the only one to contain strains from only one pathotype, i.e. APEC strains; the other clusters contained strains from several pathotypes.

As a whole, these results showed that significant differences exist in the immediate vicinity of tDNA loci between strains of different phylogenetic groups and possibly between strains of different pathotypes.

\section{DISCUSSION}

This paper describes the first large-scale PCR-based screening to identify tDNA loci in the $E$. coli genome that are generally intact and those that are preferentially associated with ECDNA regions.

In this study, an absence of amplification was taken as an indication of the presence of an ECDNA region. A negative PCR result could also have occurred because of the absence of the sequence complementary to one of the primers or because of PCR failure. The first possibility was considered unlikely: primers were designed so that they are present in the genome of four E. coli strains. Moreover, Ou et al. (2006) have shown that upstream and downstream regions of a subset of $20 \mathrm{tDNA}$ genes were highly conserved. The likelihood of PCR failure was also greatly reduced by the use of chromosomal DNA suitable for amplification of large PCR fragments as shown with primer pairs lysV1/lysV2bis or asnV2/asnW2. Additionally, control PCR reactions were performed in all experiments using chromosomal DNA from strains MG1655, CFT073 and EDL933 in parallel with 
reactions containing chromosomal DNA from the other strains studied.

The results of this study clearly indicate that a majority of tDNA loci are unlikely ECDNA insertion sites. Since ECDNA regions are frequently genomic islands associated with integrase genes, it is not surprising that, except for met $V$, our results confirm that of Williams (2002) showing that no integrase had ever been described downstream of tDNA encoding Glu, His, Gln and Met isoacceptors. While met $V$ might not be associated with genomic islands containing integrase genes as described by Williams (2002), our results indicate that it can still serve as an ECDNA insertion site as is observed in strain CFT073. In addition, Fouts (2006) recently showed that prophages, which are potential ECDNA regions, are more frequently found downstream of Arg, Leu, Thr and Ser tDNA loci: in our study these tDNA loci were found to have low integrity scores. Interestingly, although the presence of ECDNA regions downstream of phe $U$ and $p h e V$ is suggested by our results and was detected in several sequenced E. coli strains, Fouts (2006) did not find any prophage downstream of Phe tDNA.

Although several hypotheses have been made to explain the frequent association of ECDNA regions with tDNA loci (Cheetham \& Katz, 1995; Hou, 1999; Reiter et al., 1989; Saier, 1995; Williams, 2002), it is still unclear why certain tDNAs are preferred ECDNA insertion sites. We can suggest several reasons why a tDNA is not an ECDNA insertion site, although none of them can fully explain our results. First, we have shown that, with the exception of met $V$, tDNAs organized as polycistronic operons are intact in a majority of strains. We hypothesize that disruption of these loci by ECDNA insertion might prevent the processing of the RNA transcript required to form individual tRNA molecules. This would consequently affect the amount of several tRNAs and have a more severe effect on bacterial growth. Second, our data support the hypothesis that insertion of ECDNA downstream of a tDNA might affect its functionality and therefore be disadvantageous. Indeed we found that tDNAs where insertions were less frequently found had a high estimated transcription rate or encoded tRNA recognizing frequently used codons. These results may mirror the cellular requirement not to impair efficient translation by disruption of tDNA loci that encode isoacceptors present in higher amounts in the tRNA pool of the cell or recognizing frequently used codons.

To date we have no indication as to which of these criteria is the most important in determining the extent to which a tDNA can be targeted by ECDNA insertion. To strengthen our results, data concerning the affinity of one tRNA for the different anticodon it recognizes would be required to calculate the tDNA usage more precisely. Similarly, data from Ardell \& Kirsebom (2005) are only estimated transcription rates of tDNAs: availability of the actual tDNA expression level for each individual tDNA would allow more precise calculations, which might affect our conclusions. Another hypothesis that we could not test because of the lack of data in the literature is that the conditions in which a tDNA is expressed can influence its role as an ECDNA insertion site. In that respect, it is worth mentioning that four tDNAs encoding tRNA ${ }^{\text {Leu }}$ were found to be differently expressed depending on the growth conditions (Dobrindt \& Hacker, 2001; Rowley et al., 1993) and that only the transcription of the leuX tDNA, which had the lowest score of all leu tDNA loci, was not growth phase regulated (Rowley et al., 1993). Whether this is also the case for other tDNA loci remains to be elucidated.

The flanking sequence context of each tDNA is also an important determinant. Several tDNA loci (either monocistronic or polycistronic) have been shown to be cotranscribed with downstream genes (Bosl \& Kersten, 1991; Fournier \& Ozeki, 1985; Li \& Deutscher, 2002). Disruption of such transcriptional units could therefore be detrimental to the bacteria. For example, it is not surprising that the $\operatorname{thr} T$ tDNA had a high score since it is co-transcribed with $t u f B$, the product of which is an important element of the translation machinery and whose deletion in certain genetic backgrounds significantly reduces the growth rate of $E$. coli (Lee et al., 1981; Schnell et al., 2003).

The local DNA structure of a tDNA, or the binding of proteins modifying the DNA conformation, might also influence the ability of a tDNA to serve as an ECDNA insertion target. Indeed, the activity of integrase genes found in several genomic islands and involved in genomic islands mobility is likely to depend upon the DNA structure (Cali et al., 2004; Hochhut et al., 2006; Lesic et al., 2004; Pribil \& Haniford, 2003; Radman-Livaja et al., 2006). Interestingly, several tDNA loci with high scores such as alaX, glyY, lysQ, proM, thrT, valW or $g \ln X$ had DNA curvature profiles different from those of a number of loci with low scores such as asn loci, pheU, metV, pheV and serW. In these latter cases, the DNA curvature was found to sharply decrease inside the tDNA locus and then sharply increase at the end of the locus (see Supplementary Fig. S2 - curvature data are from Pedersen et al., 2000). Whether this is linked to the use of tDNA loci as ECDNA insertion hot-spots remains to be clarified.

A major finding of this work is the fact that the tDNA pattern of one strain is related to its phylogenetic (ECOR) group. The MFCA analysis performed in this study separated B2 strains from strains of the three other ECOR groups as well as strains from ECOR groups A and B1 (Fig. 2a, b). This was further illustrated by the hierarchical clustering performed from the MFCA results (Fig. 3). This distinction based on the ECOR group is reminiscent of the hypothesis made by Escobar-Paramo et al. (2004), who showed that the genetic background of strains belonging to the B2 ECOR group might be a critical player in the acquisition of ExPEC virulence factors. Since tDNAs are ECDNA insertion hot-spots, the tDNA pattern of one strain is also likely to have an influence on the acquisition/loss of ECDNA regions and could explain the specific properties of B2 strains, including their pathogenic properties (Picard 
et al., 1999). Furthermore, ExPEC strains mostly belong to the B2 group and to a lesser extent to the D group (Picard et al., 1999; Sannes et al., 2004); it is therefore possible that tDNA combinations preferentially associated with $\mathrm{B} 2$ or D strains detected in our study are necessary for the expression of the full virulence of these strains.

The third cluster of strains identified in the hierachical clustering was found to be very heterogeneous. Actually, Escobar et al. (2004) identified two subgroups (groups C and E) that are not discriminated by the PCR described by Clermont et al. (2000) used in this study. It is therefore possible that the heterogeneity of cluster 3 is linked to the fact that it contains strains from groups $\mathrm{C}$ and $\mathrm{E}$.

Our results also indicate that some differences may exist between strains of different pathotypes. Analysis of our results unravelled some differences between APEC and human ExPEC strains. Among these differences, we found that the ser $W$ tDNA was a preferred ECDNA insertion hotspot in APEC but not in other ExPEC strains. It will therefore be interesting to characterize the sequence present downstream of serW in these APEC strains and investigate whether it can encode host specificity factors.

Complementary to these conclusions, our data give an indication as to which tDNAs can serve as ECDNA insertion sites. Chouikha et al. (2006) recently used the lack of amplification of a PCR product at selC in an APEC strain to identify and characterize a new genomic island (AGI-3). A similar PCR-based strategy was designed by Ou et al. (2006) to search for new genomic islands and tested in silico, thus validating the PCR-based approach for the identification of new genomic islands. The $15 \mathrm{tDNA}$ loci that we have identified as frequent insertion sites (score $<47$ ) were found by these authors as frequent genomic island insertion sites.

In conclusion, this article describes the first large-scale study of the sequence context of tDNA loci in different E. coli isolates. We show that only a few tDNA loci are potential chromosomal insertion sites for large ECDNA regions. In fact, our results show that a majority of tDNAs are rarely sites of ECDNA insertion and provide evidence that this might be related to the organization of the loci, to their transcription rate and to their usage. Most interestingly, we demonstrated that the tDNA organization of a particular strain is related to the phylogenetic group it belongs to.

\section{ACKNOWLEDGEMENTS}

This project was funded by the EC under FP5 (COLIRISK - no. QLK2CT-2002-00944). Work of the Würzburg group was also supported by the Deutsche Forschungsgemeinschaft (SFB479, Teilprojekt A1). We thank T. Whittam for providing us with ECOR strains, P. Gilot for critical reading of the manuscript and $\mathrm{M}$. Répérant for ECOR grouping of avian E. coli strains.

\section{REFERENCES}

Anton, A. I., Martinez-Murcia, A. J. \& Rodriguez-Valera, F. (1998). Sequence diversity in the $16 \mathrm{~S}-23 \mathrm{~S}$ intergenic spacer region (ISR) of the rRNA operons in representatives of the Escherichia coli ECOR collection. J Mol Evol 47, 62-72.

Ardell, D. H. \& Kirsebom, L. A. (2005). The genomic pattern of tDNA operon expression in E. coli. PLoS Comput Biol 1, e12.

Asai, T., Condon, C., Voulgaris, J., Zaporojets, D., Shen, B., Al-Omar, M., Squires, C. \& Squires, C. L. (1999). Construction and initial characterization of Escherichia coli strains with few or no intact chromosomal rRNA operons. J Bacteriol 181, 3803-3809.

Blattner, F. R., Plunkett, G., 3rd, Bloch, C. A., Bloch, C. A., Perna, N. T., Burland, V., Riley, M., Collado-Vides, J., Glasner, J. D. \& other authors (1997). The complete genome sequence of Escherichia coli K-12. Science 277, 1453-1474.

Bosl, M. \& Kersten, H. (1991). A novel RNA product of the tyrT operon of Escherichia coli. Nucleic Acids Res 19, 5863-5870.

Boyd, E. F. \& Hartl, D. L. (1998). Chromosomal regions specific to pathogenic isolates of Escherichia coli have a phylogenetically clustered distribution. J Bacteriol 180, 1159-1165.

Cali, S., Spoldi, E., Piazzolla, D., Dodd, I. B., Forti, F., Deho, G. \& Ghisotti, D. (2004). Bacteriophage P4 Vis protein is needed for prophage excision. Virology 322, 82-92.

Cheetham, B. F. \& Katz, M. E. (1995). A role for bacteriophages in the evolution and transfer of bacterial virulence determinants. Mol Microbiol 18, 201-208.

Chouikha, I., Germon, P., Brée, A., Gilot, P., Moulin-Schouleur, M. \& Schouler, C. (2006). A selC-associated genomic island of the extraintestinal avian pathogenic Escherichia coli strain BEN2908 is involved in carbohydrate uptake and virulence. J Bacteriol 188, 977-987.

Clermont, O., Bonacorsi, S. \& Bingen, E. (2000). Rapid and simple determination of the Escherichia coli phylogenetic group. Appl Environ Microbiol 66, 4555-4558.

Condon, C., Liveris, D., Squires, C., Schwartz, I. \& Squires, C. L. (1995). rRNA operon multiplicity in Escherichia coli and the physiological implications of $r r n$ inactivation. J Bacteriol 177, $4152-4156$.

Dho, M. \& Lafont, J. P. (1984). Adhesive properties and iron uptake ability in Escherichia coli lethal and nonlethal for chicks. Avian Dis 28, 1016-1025.

Dobrindt, U. \& Hacker, J. (2001). Regulation of tRNA5Leu-encoding gene $\operatorname{leuX}$ that is associated with a pathogenicity island in the uropathogenic Escherichia coli strain 536. Mol Genet Genomics 265, 895-904.

Dobrindt, U., Blum-Oehler, G., Hartsch, T., Gottschalk, G., Ron, E. Z., Fünfstück, R. \& Hacker, J. (2001). S-Fimbria-encoding determinant $s f a(I)$ is located on pathogenicity island III(536) of uropathogenic Escherichia coli strain 536. Infect Immun 69, 4248-4256.

Dobrindt, U., Hochhut, B., Hentschel, U. \& Hacker, J. (2004). Genomic islands in pathogenic and environmental microorganisms. Nat Rev Microbiol 2, 414-424.

Dozois, C. M. \& Curtiss, R., 3rd (1999). Pathogenic diversity of Escherichia coli and the emergence of 'exotic' islands in the gene stream. Vet Res 30, 157-179.

Escobar-Paramo, P., Clermont, O., Blanc-Potard, A. B., Bui, H., Le Bouguénec, C. \& Denamur, E. (2004). A specific genetic background is required for acquisition and expression of virulence factors in Escherichia coli. Mol Biol Evol 21, 1085-1094.

Fournier, M. J. \& Ozeki, H. (1985). Structure and organization of the transfer ribonucleic acid genes of Escherichia coli K-12. Microbiol Rev 49, 379-397. 
Fouts, D. E. (2006). Phage_Finder: automated identification and classification of prophage regions in complete bacterial genome sequences. Nucleic Acids Res 34, 5839-5851.

Hacker, J., Hentschel, U. \& Dobrindt, U. (2003). Prokaryotic chromosomes and disease. Science 301, 790-793.

Hayashi, T., Makino, K., Ohnishi, M., Kurokawa, K., Ishii, K., Yokoyama, K., Han, C. G., Ohtsubo, E., Nakayama, K. \& other authors (2001). Complete genome sequence of enterohemorrhagic Escherichia coli $\mathrm{O} 157: \mathrm{H} 7$ and genomic comparison with a laboratory strain K-12. DNA Res 8, 11-22.

Hochhut, B., Wilde, C., Middendorf, B., Dobrindt, U., Brzuszkiewicz, E., Gottschalk, G., Carniel, E. \& Hacker, J. (2006). Role of pathogenicity island-associated integrases in the genome plasticity of uropathogenic Escherichia coli strain 536. Mol Microbiol 61, 555-595.

Hou, Y. M. (1999). Transfer RNAs and pathogenicity islands. Trends Biochem Sci 24, 295-298.

Kaper, J. B., Nataro, J. P. \& Mobley, H. L. (2004). Pathogenic Escherichia coli. Nat Rev Microbiol 2, 123-140.

King, T. C., Sirdeskmukh, R. \& Schlessinger, D. (1986). Nucleolytic processing of ribonucleic acid transcripts in procaryotes. Microbiol Rev 50, 428-451.

Lecointre, G., Rachdi, L., Darlu, P. \& Denamur, E. (1998). Escherichia coli molecular phylogeny using the incongruence length difference test. Mol Biol Evol 15, 1685-1695.

Lee, J. S., An, G., Friesen, J. D. \& Fill, N. P. (1981). Location of the tufB promoter of $E$. coli: cotranscription of $t u f B$ with four transfer RNA genes. Cell 25, 251-258.

Lesic, B., Bach, S., Ghigo, J. M., Dobrindt, U., Hacker, J. \& Carniel, E. (2004). Excision of the high-pathogenicity island of Yersinia pseudotuberculosis requires the combined actions of its cognate integrase and Hef, a new recombination directionality factor. $\mathrm{Mol}$ Microbiol 52, 1337-1348.

Li, Z. \& Deutscher, M. P. (1996). Maturation pathways for E. coli tRNA precursors: a random multienzyme process in vivo. Cell $\mathbf{8 6}$, 503-512.

Li, Z. \& Deutscher, M. P. (2002). RNase E plays an essential role in the maturation of Escherichia coli tRNA precursors. RNA 8, 97-109.

Morl, M. \& Marchfelder, A. (2001). The final cut. The importance of tRNA 3'-processing. EMBO Rep 2, 17-20.

Ochman, H. \& Selander, R. K. (1984). Standard reference strains of Escherichia coli from natural populations. J Bacteriol 157, 690-693.

Ou, H. Y., Chen, L. L., Lonnen, J., Chaudhuri, R. R., Thani, A. B., Smith, R., Garton, N. J., Hinton, J., Pallen, M. \& other authors (2006). A novel strategy for the identification of genomic islands by comparative analysis of the contents and contexts of tRNA sites in closely related bacteria. Nucleic Acids Res 34, e3.

Pedersen, A. G., Jensen, L. J., Brunak, S., Staerfeldt, H. H. \& Ussery, D. W. (2000). A DNA structural atlas for Escherichia coli. J Mol Biol 299, 907-930.

Perna, N. T., Plunkett, G., 3rd, Burland, V., Mau, B., Glasner, J. D., Rose, D. J., Mayhew, G. F., Evans, P. S., Gregor. J. \& other authors (2001). Genome sequence of enterohaemorrhagic Escherichia coli O157: H7. Nature 409, 529-533.
Picard, B., Garcia, J. S., Gouriou, S., Duriez, P., Brahimi, N., Bingen, E., Elion, J. \& Denamur, E. (1999). The link between phylogeny and virulence in Escherichia coli extraintestinal infection. Infect Immun 67, 546-553.

Pribil, P. A. \& Haniford, D. B. (2003). Target DNA bending is an important specificity determinant in target site selection in $\operatorname{Tn} 10$ transposition. J Mol Biol 330, 247-259.

Radman-Livaja, M., Biswas, T., Ellenberger, T., Landy, A. \& Aihara, H. (2006). DNA arms do the legwork to ensure the directionality of lambda site-specific recombination. Curr Opin Struct Biol 16, 42-50.

Redford, P. \& Welch, R. A. (2002). Extraintestinal Escherichia coli as a model system for the study of pathogenicity islands. Curr Top Microbiol Immunol 264, 15-30.

Reiter, W. D., Palm, P. \& Yeats, S. (1989). Transfer RNA genes frequently serve as integration sites for prokaryotic genetic elements. Nucleic Acids Res 17, 1907-1914.

Ron, E. Z. (2006). Host specificity of septicemic Escherichia coli: human and avian pathogens. Curr Opin Microbiol 9, 1-5.

Rowley, K. B., Elford, R. M., Roberts, I. \& Holmes, W. M. (1993). In vivo regulatory responses of four Escherichia coli operons which encode leucyl-tRNAs. J Bacteriol 175, 1309-1315.

Saier, M. H., Jr (1995). Differential codon usage: a safeguard against inappropriate expression of specialized genes? FEBS Lett 362, 1-4.

Sannes, M. R., Kuskowski, M. A., Owens, K., Gajewski, A. \& Johnson, J. R. (2004). Virulence factor profiles and phylogenetic background of Escherichia coli isolates from veterans with bacteremia and uninfected control subjects. J Infect Dis 190, 2121-2128.

Schnell, R., Abdulkarim, F., Kalman, M. \& Isaksson, L. A. (2003). Functional EF-Tu with large C-terminal extensions in an E. coli strain with a precise deletion of both chromosomal tuf genes. FEBS Lett 538, 139-144.

Selander, R. K., Caugant, D. \& Whittam, T. S. (1987). Genetic structure and variation in natural populations of Escherichia coli. In Escherichia coli and Salmonella typhimurium: Cellular and Molecular Biology, pp. 1625-1648. Edited by F. Neidhardt and others. Washington, DC: American Society for Microbiology.

Sharp, P. A. \& Li, W. (1986). Codon usage in regulatory genes in Escherichia coli does not reflect selection for 'rare' codons. Nucleic Acids Res 14, 7737-7749.

Stordeur, P., Marlier, D., Blanco, J., Oswald, E., Biet, F., Dho-Moulin, M. \& Mainil, J. (2002). Examination of Escherichia coli from poultry for selected adhesin genes important in disease caused by mammalian pathogenic E. coli. Vet Microbiol 84, 231-241.

Welch, R. A., Burland, V., Plunkett, G., 3rd, Redford, P., Roesch, P., Rasko, D., Buckles, E. L., Liou, S. R., Boutin, A. \& other authors (2002). Extensive mosaic structure revealed by the complete genome sequence of uropathogenic Escherichia coli. Proc Natl Acad Sci U S A 99, 17020-17024.

Williams, K. P. (2002). Integration sites for genetic elements in prokaryotic tRNA and tmRNA genes: sublocation preference of integrase subfamilies. Nucleic Acids Res 30, 866-875.

Edited by: D. W. Ussery 